\title{
Transport suppression in polymer-doped zigzag carbon nanotubes
}

\author{
D. Grimm, ${ }^{1}$ A. Latgé, ${ }^{1, *}$ R. B. Muniz, ${ }^{1}$ and M. S. Ferreira ${ }^{2}$ \\ ${ }^{1}$ Instituto de Física, Universidade Federal Fluminense, 24210-340 Niterói-Rio de Janeiro, Brazil \\ ${ }^{2}$ Physics Department, Trinity College Dublin, Dublin, Ireland
}

(Received 3 October 2004; published 21 March 2005)

\begin{abstract}
The conductance of zigzag carbon nanotubes in the presence of grafted polymers attached to their walls is investigated. A theoretical study of the dependence of the transport on the nature of the molecule and on the concentration of attached structures is presented. The attached structures are shown to lead to the formation of resonant states trapping the electronic carriers and suppressing the conductance. An exponential decay of the conductance with increasing concentration of attached molecules marks the ballistic regime while a weaker decay dependence highlights a crossover to a diffusivelike electronic behavior.
\end{abstract}

DOI: 10.1103/PhysRevB.71.113408

The use of individual molecules as functional electronic devices is currently in focus due to the miniaturization strategies pursued by the electronic and computer industries. Molecular electronics has become a field of research which is progressing at a fast pace thanks to recent developments in nanotechnology. Advances in physical and chemical sciences have been crucial in this field. On the physics side, the usual down-size approach of working with systems of continuing smaller dimensions has made possible the visualization and manipulation of single atoms and molecules, and modern spectroscopic techniques can now probe the fascinating quantum features of molecular orbitals. ${ }^{1}$ Chemists, on the other side, are presently capable of synthesizing larger molecules in a controlled fashion and of producing compounds with tailor-made chemical and physical properties.

Carbon nanotubes (CN's) belong to a special class of molecules that are suitable for electronics. They are found in a variety of geometries with unique transport and electronic features. The combination of interesting transport properties, mechanical strength, and flexibility makes them ideal candidates for building blocks of nanoscale electronic devices. In fact, several CN-based devices have already been proposed, such as logic circuits ${ }^{2}$ and field-effect ${ }^{3}$ and single-electron transistors, ${ }^{4}$ to name but a few. The construction of more complex structures based on CN's is crucial to expand their applicability. There is growing interest in the possibility of controlling the nature, size, and concentration of foreign objects that can interact with nanotubes. Foreign attachments to the walls of a $\mathrm{CN}$ can occur as a result of van der Waals interactions and covalent bonding. ${ }^{5,6}$ One of the reasons for controlling the attachment of molecules or nanoparticles to the walls of CNs is that these chemical bonds may be used to alter the interaction of the $\mathrm{CN}$ with other atoms, polymeric molecules, or even other tubes. Moreover, the functionalized tube may change its mechanical and electronic properties due to the foreign object. In fact, potential use of CN's as chemical sensors and nanometer-scale electronic devices has already been envisaged. ${ }^{7}$ Furthermore, recent reports point to the use of chemically modified carbon tubes as a substrate for neuronal growth. ${ }^{8}$

With such a motivation, we investigate in this paper how the transport properties of a $\mathrm{CN}$ are affected by the absorption of a foreign structure to its walls. Our objective is to
PACS number(s): 73.22.-f, 73.23.-b, 73.63.-b

study not the detailed mechanisms of the binding process, but the effect the integrated structure brings to the quantum conductance of the system. This certainly depends on the nature of the attaching object. Polymers are one of the many different structures that can interact with a $\mathrm{CN}$. Those long quasi-one-dimensional molecules can adhere to a nanotube in different ways, depending on the type of polymer and on the solutions in which they are initially immersed. Polymer strands coiling around carbon nanotubes have been identified and seem to be responsible for a dramatic degree of mechanical reinforcement in composite structures. ${ }^{9}$ In another geometry, polymers can have their ends attached to the nanotube. When covalently bound, the effect of this interaction on the transport properties of the system is reasonably strong. Rather than interacting with a large portion of the carbon atoms, these so-called grafted polymers cause a more localized effect at the atomic sites to which they stick. This is a simpler system to model and is the reference geometry for our studies, namely, finite one-dimensional molecules with one of their ends bound to the walls of an infinitely long $\mathrm{CN}$. The inset of Fig. 1 shows an atomic representation of one of the simple structures considered here.

The quantum conductance $\Gamma$ is calculated by the KuboLandauer formula expressed in terms of single-particle Green functions (GF), which are the key quantities in our formalism. ${ }^{10}$ In a metallic $\mathrm{CN}$ with ideal electrical contacts one finds perfect transmission characterized by two units of quantum conductance $\Gamma=2 \Gamma_{0}=4 e^{2} / h \cdot{ }^{11,12}$ How the transport properties are affected by the molecular attachments can be answered by investigating how the GF matrix elements depend on fundamental parameters, such as the concentration of attached molecules, the coupling between those molecular structures and the tube, the molecular sizes, and their specific energy levels. Although high-precision ab initio techniques have been used to study how the electronic structure of a $\mathrm{CN}$ is affected by the introduction of foreign atoms, ${ }^{13}$ they are limited to a few attachments and are currently unable to treat highly disordered environments such as the ones we want to investigate. Simple models for the electronic structure are then quite appropriate for the present investigation.

To illustrate the model calculation adopted here we consider first the disconnected system consisting of a $\mathrm{CN}$ and a finite-sized molecule taken separately. The electronic struc- 


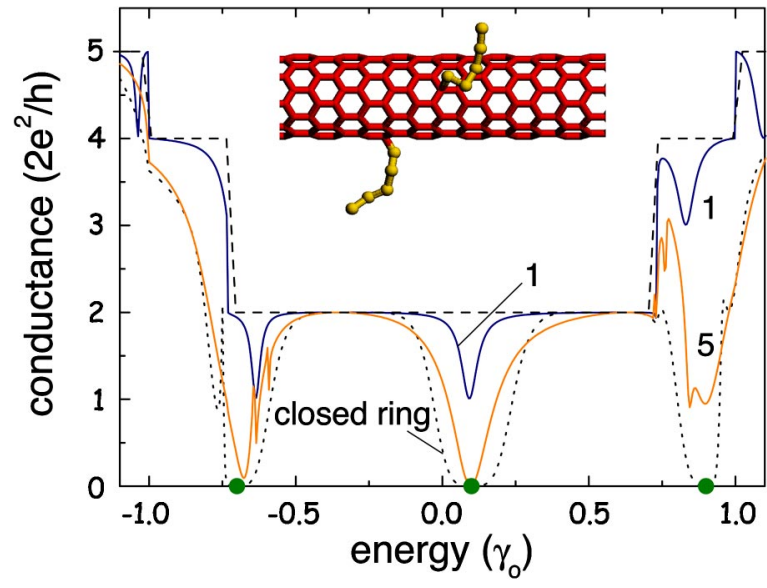

FIG. 1. (Color online) Conductance of a $(6,0) \mathrm{CN}$ with molecules (finite chains with five atoms) attached to its walls, as a function of the Fermi energy. The two solid curves correspond to the cases of one and five attached molecules, whereas the dotted line is for a closed ring configuration and the dashed one for a pure $\mathrm{CN}$. Solid circles at the energy axis mark the electronic eigenvalues of the attached chain. Energies are given in units of the hopping energy $\left(\gamma_{0}=1\right)$ and the molecule parameters are chosen as $\epsilon_{m}=0.1$ and $\gamma_{m}=0.8 ; \gamma_{c}=1$.

ture of the former is known to be well described by a singleband tight-binding model, ${ }^{14,15}$ at least for energies close to the Fermi level. We represent the molecule by a finite-sized linear chain of atoms using the same model. In what follows, the diagonal and off-diagonal energies of the isolated parts are taken as $\epsilon_{0}$ and $\gamma_{0}$ for the $\mathrm{CN}$ and $\epsilon_{m}$ and $\gamma_{m}$ for the molecule. All energy parameters are given in terms of $\gamma_{0}$, and we choose our energy origin at $\epsilon_{0}=0$. The GF of the disconnected system is represented by $\mathcal{G}_{j, j^{\prime}}$, where $j$ and $j^{\prime}$ label atomic sites. Clearly, $\mathcal{G}_{j, j^{\prime}}=0$ if $j$ and $j^{\prime}$ refer to sites on either part of the disconnected system, because in this case there is no electronic propagation between the parts. We connect the molecule to the tube by switching on the electronic hopping $\left(\gamma_{c}\right)$ between the parts. For simplicity, we assume that only the end atom of the molecule is connected to a single site of the $\mathrm{CN}$. It is worth mentioning that this is not a fundamental assumption and that contact with more sites can be easily included.

An arbitrary GF matrix element of the connected system is given by

$$
G_{j, j^{\prime}}(E)=\mathcal{G}_{j, j^{\prime}}+\mathcal{G}_{j, 0} \gamma_{c}\left(1-\mathcal{G}_{1,1} \gamma_{c} \mathcal{G}_{0,0} \gamma_{c}\right)^{-1} \mathcal{G}_{1,1} \gamma_{c} \mathcal{G}_{0, j^{\prime}},
$$

where the sites 0 and 1 are the connecting sites on the tube and on the end atom of the molecule, respectively, and $j$ and $j^{\prime}$ are atomic sites belonging to the tube. In this way, the GF of the connected structure is written in terms of that for the disconnected system. One immediate consequence of the equation above is that the effect of the molecular attachment on the $\mathrm{CN}$ is explicitly displayed through the surface Green function of the molecular structure $\left(\mathcal{G}_{1,1}\right)$. It is clear that small values of $\mathcal{G}_{1,1}$ lead to a regime of molecular transparency, in which the electron propagation is exclusively deter- mined by the unperturbed GF of the isolated $\mathrm{CN}$. On the opposite limit, $\mathcal{G}_{1,1}$ also carries information about the resonant states of the molecule, leading to a divergence on the second term of Eq. (1). It is worth mentioning that these states correspond to the eigenstates of the isolated molecule. In this scenario, the conductance is expected to be strongly affected by the molecular attachments which may act as a perfect trap for the electronic carriers. In this resonant regime the conductance is expected to be partially suppressed.

The conductances of a metallic $(6,0) \mathrm{CN}$ as functions of the Fermi energy are shown in Fig. 1, for the cases where one and five molecules are attached to its wall. The attached molecules are atomic chains consisting of five atoms, and are characterized by $\epsilon_{m}=0.1, \gamma_{m}=0.8$, and $\gamma_{c}=1$. The dashed line displays the conductance for an isolated tube (disconnected $\mathrm{CN}$ ) exhibiting the two electronic channels for energies around the Fermi level $\epsilon_{F}=0$, a characteristic result of pure CN's. Full circles in the energy axis denote the linear chain eigenvalues obtained from the corresponding surface GF. For the case of one single attached molecule, although no complete resonant regime is found, the conductance is partially suppressed at the energy values for which the electrons are trapped in the molecule eigenstates. This is due to the fact that, unlike what happens with a complete onedimensional case, ${ }^{16}$ there are alternative paths the electron can take to avoid the connecting site on the tube. For more than one attached molecule, one finds a complete suppression of the transport at the central eigenvalue, while for the other ones (close to $\epsilon=0.9$ and -0.7 ) the extension of the conductance reduction is governed by the number of grafted molecules. For the sake of illustration, a limiting regime is also considered in which a set of identical chains are attached to the $\mathrm{CN}$ wall, forming a closed ring of grafted molecules. In this case, a carrier cannot travel past the structure without hitting one of those polymerized sites. This is shown in the figure by the dotted line, where the conductance vanishes at those energy values that coincide with the molecule resonant states. According with Eq. (1), we can identify energies for which the grafted molecules are entirely transparent to the electronic carriers.

More realistically, the molecules are expected to adhere (randomly) to the $\mathrm{CN}$ walls. One of the advantages of the present model is its ability to incorporate such kind of disorder within the Green function formalism in real space. We consider a large section of the CN containing 100-200 carbon rings to which molecules may be randomly attached. By summing over a large number of configurations (500-2000) we are able to calculate the average conductance for a given concentration of attached molecules. An illustrative result is shown in Fig. 2 which displays the conductance of a $(6,0)$ $\mathrm{CN}$ with percentages equal to $1 \%$ (solid curve) and $10 \%$ (dashed line) of connected molecules for a relatively low value of the coupling $\gamma_{c}=0.3$. The calculated results are for molecules with 24 atoms, $\epsilon_{m}=0.1$ and $\gamma_{m}=0.8$. The electronic eigenvalues of the isolated molecule are indicated by full circles on the energy axis. It is clear that in the case of randomly distributed molecules the conductance is also suppressed at those energies that coincide with the isolate chain eigenvalues. Likewise, transparent regimes are also present in this case. For higher concentrations the conductance re- 


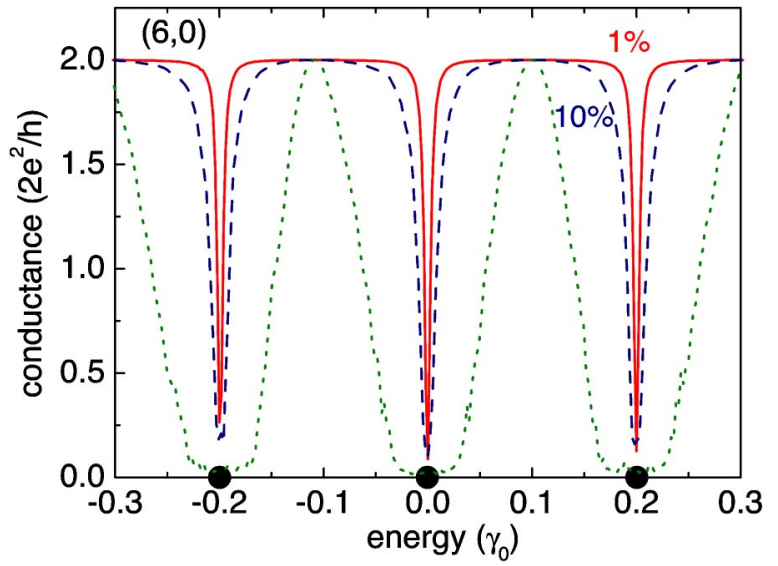

FIG. 2. (Color online) Conductances as functions of energy for a $(6,0) \mathrm{CN}$ grafted with 24-atom linear chain molecules. Solid circles represent the electronic eigenenergies of the isolated chains. Results are for $1 \%$ (solid curve) and 10\% (dashed line) molecular concentrations; $\gamma_{c}=0.3, \epsilon_{m}=0.1$, and $\gamma_{m}=0.8$. Dotted line is for $\gamma_{c}=0.9$ and molecular concentration $10 \%$.

ductions occur in extended regions around the isolated molecule eigenvalues. The effect is more pronounced for higher tube-molecule coupling values, as shown by the dotted line for $\gamma_{c}=0.9$ and a percentage equal to $10 \%$, as discussed in more detail below.

The conductance suppression is also shown in Fig. 3(a) where $\Gamma$ (calculated for $\epsilon_{F} \approx 0.2$ ) is plotted as a function of molecule concentrations for a $(9,0)$ metallic tube. We identify two distinct conductance regimes, separated by a transition region. $\Gamma$ decays exponentially for very low concentrations $(x<1 \%)$, and decreases almost linearly for $x>50 \%$. The inset highlights the exponential decaying regime for two distinct molecule sizes (24 and 115 atoms). We have also calculated the conductance distributions for different molecule concentrations, and the results are depicted in Fig. 3 (b). The corresponding average conductances are indicated by wide tick marks in the $\hat{x}$ axis. We notice that for $x=5 \%$ and $27 \%$ the distributions are log-normal, indicating a transition from ballistic to insulating behavior. ${ }^{17}$ For high concentrations the conductance variation is strongly reduced, as
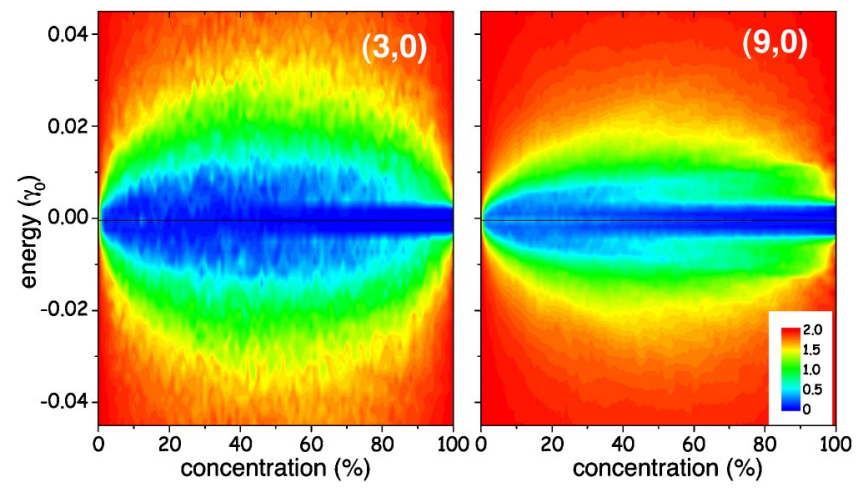

FIG. 4. (Color online) Gray-scale maps of the conductance as functions of the coverage percentage of attached molecules and the Fermi level, written in terms of the tube hopping $\gamma_{0}$. The results are for molecules described by finite 24-atom chains with $\epsilon_{m}=0.1$ and $\gamma_{m}=0.8$. Results are for $(3,0)$ and $(9,0)$ tubes at the left and right parts of the figure, respectively.

illustrated by the rightmost panel of Fig. 3(b). This indicates that most of the free carrier paths are already blocked and the system becomes rather insensitive to statistical distributions and further molecule aggregations.

The evolution of the conductance suppression is depicted in Fig. 4 with a gray-scale (on-line colored) two-dimensional plot for energies around one of the chain's eigenvalues. The diagrams show the conductance as a function of both the molecular concentration and the Fermi energy for $(3,0)$ and $(9,0)$ CN's. Well-defined conductance gaps centered at the molecule eigenvalues are clearly visible for concentrations $\geq 40 \%$ [black (dark-blue) region] in contrast to the higher disordered region in which variable wider gaps are formed in the vicinity of the eigenenergy. Analogously to the previously discussed case, the conductance suppression is not as abrupt for the $(9,0)$ tube due to the larger number of paths the carriers can take in avoiding the random scatterers. Previous calculations for armchair CN's in the presence of disorder have also shown that the electronic mean free path increases with the tube diameter. ${ }^{11}$ The extremely thin $(3,0)$ tube considered here shows a greater conductance suppression in the vicinity of the polymer eigenvalues, as expected.
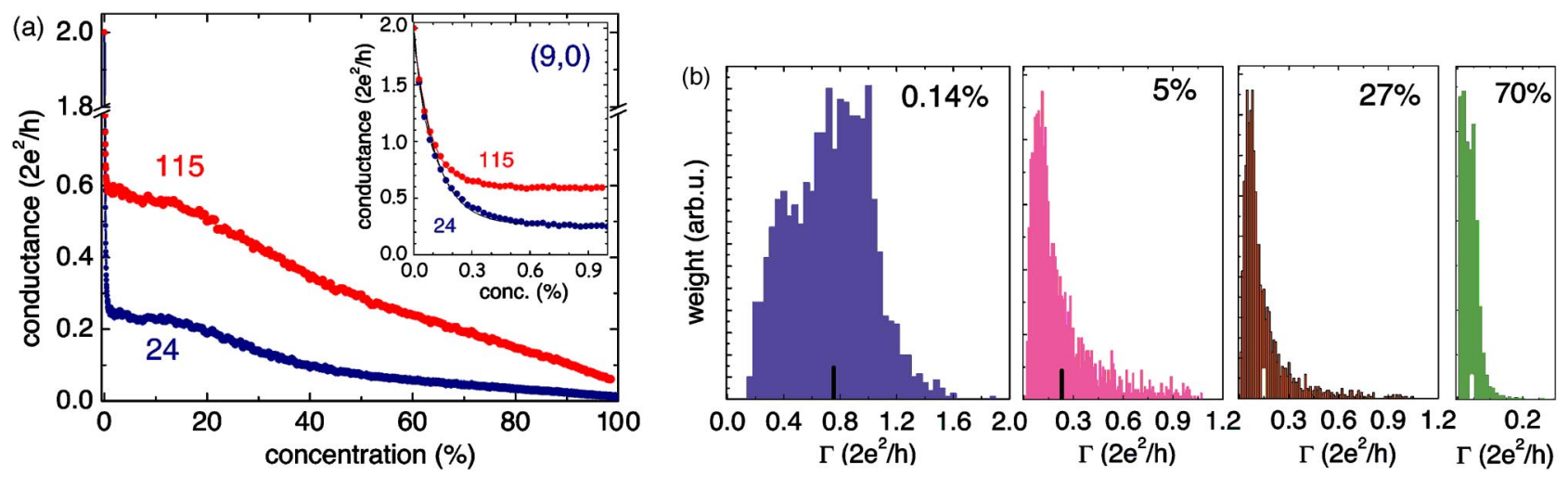

FIG. 3. (Color online) (a) Conductance decay as a function of the concentration of molecules (24- and 115-atom chains) attached to a $(9,0) \mathrm{CN}$, at one of the eigenvalues of the chains. Inset shows the exponential decay for low concentrations. (b) Conductance distribution for a $(9,0) \mathrm{CN}$ with $0.14 \%, 5 \%, 27 \%$, and $70 \%$ of the $\mathrm{CN}$ sites covered by polymers $\left(\gamma_{c}=0.3, \epsilon_{m}=0.1\right.$, and $\left.\gamma_{m}=0.8\right)$. 


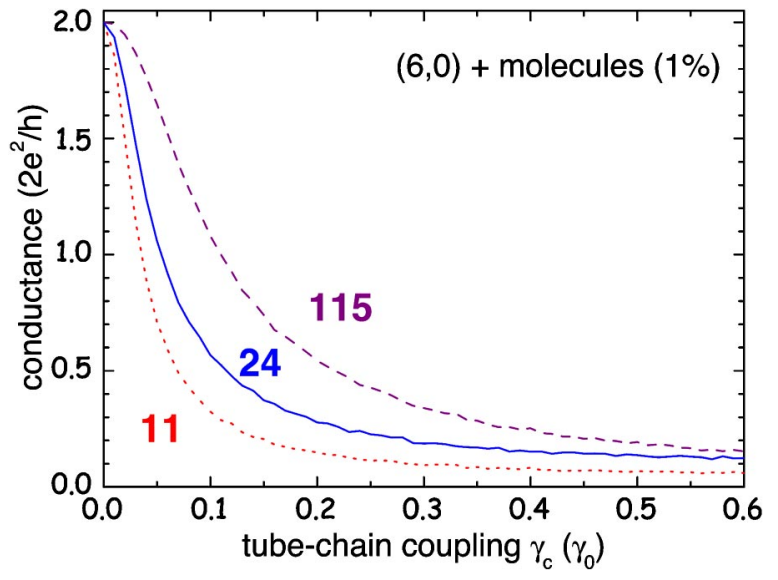

FIG. 5. (Color online) Conductance of a $(6,0) \mathrm{CN}$ with a fixed molecular concentration of $1 \%$ as a function of the coupling energy between the $\mathrm{CN}$ and the chain and at a Fermi energy coincident with the central eigenvalue of the attached chain. Distinct lengths are considered for the attached molecules $\left(\epsilon_{m}=0.1, \gamma_{m}=0.8\right.$, and $\left.\gamma_{c}=0.3\right)$.

The results discussed above are for molecules that are weakly coupled to the nanotube, for instance, by means of a van der Waals type of interaction. Covalently bound molecules, however, will certainly be much more strongly coupled to the $\mathrm{CN}$ wall. A detailed description of such a situation requires more involved calculations based on first principles. Nevertheless, it is instructive to investigate the effect of the coupling strength on the conductance by varying $\gamma_{c}$, as well as the role played by the molecule size.

Conductance results, calculated at one of the chain eigen- values, as functions of $\gamma_{c}$, are shown in Fig. 5 for different chain sizes (consisting of 11, 24, and 115 atoms). As expected, the conductance suppression is more pronounced for stronger couplings. Moreover, $\Gamma$ decays exponentially with $\gamma_{c}$, and the decaying rate is inversely proportional to the size of the attached chain. This effect is similar to the one found for the conductance dependence on the concentration of attached molecules [inset of Fig. 3(a)]. As the amount of the resonant modes increases, the weight of the corresponding eigenvalues of the finite grafted chains in the transport decreases. One may expect, therefore, that a better statistical description of the randomly distribution should also take into account molecules of different sizes attached in the same model.

Summing up, we have calculated the effects on the conductance of polymerlike structures grafted on the walls of CNs. The simple tight-binding model adopted enables us to treat appropriately the disordered system defined by the random distribution of the attached chains. By calculating the conductance we found a transport suppression at particular energy values corresponding to the resonant chain states. One clearly notices different electronic transport regimes depending essentially on the concentration of attached molecules. This theoretical study should be helpful, for instance, in the analysis of the nature of the foreign structures that may conveniently modulate transport responses.

This work was partially supported by the Brazilian Agencies $\mathrm{CNPq}$ and CAPES, Instituto do Milênio para Nanociências/MCT/Brazil, and PRONEX-CNPq-FAPERJ Grant No. 171.168-2003. M.S.F. acknowledges the financial support of Science Foundation Ireland and Enterprise Ireland. We also thank A.T. Costa for useful discussions.
*Electronic address: latge@if.uff.br

${ }^{1}$ K. A. Williams, P. T. M. Veenhuizen, B. G. de la Torre, R. Eritja, and C. Dekker, Nature (London) 420, 761 (2002).

${ }^{2}$ A. Bachtold, P. Hadley, T. Nakanishi, and C. Dekker, Science 294, 1317 (2001).

${ }^{3}$ S. Tans, A. R. M. Verschueren, and C. Dekker, Nature (London) 393, 49 (1998).

${ }^{4}$ H. W. C. Postma, T. F. Teepen, Z. Yao, M. Grifoni, and C. Dekker, Science 293, 76 (2001).

${ }^{5}$ J. Chen, M. A. Hamon, H. Hu, Y. Chen, A. M. Rao, P. C. Eklund, and R. C. Haddon, Science 282, 95 (1998).

${ }^{6}$ R. J. Baierle, S. B. Fagan, R. Mota, A. J. R. da Silva, and A. Fazzio, Phys. Rev. B 64, 085413 (2001); 67, 033405 (2003).

${ }^{7}$ P. G. Collins, K. Bradley, M. Ishigami, and A. Zettl, Science 287, 1801 (2000).

${ }^{8} \mathrm{H}$. Hu, Y. Ni, V. Montana, R. C. Haddon, and V. Parpura, Nano Lett. 4, 507 (2004).
${ }^{9}$ J. N. Coleman and M. S. Ferreira, Appl. Phys. Lett. 84, 798 (2004).

${ }^{10}$ C. G. Rocha, T. G. Dargam, and A. Latgé, Phys. Rev. B 65, 165431 (2002); A. Latgé, D. C. Marcucci, and M. V. Tovar Costa, Physica E (Amsterdam) 13, 12624 (2002).

${ }^{11}$ C. T. White and T. N. Todorov, Nature (London) 393, 240 (1998).

${ }^{12}$ C. Dekker, Phys. Today 52 (5), 22 (1999).

${ }^{13}$ S. Latil, S. Roche, D. Mayou, and J.-C. Charlier, Phys. Rev. Lett. 92, 256805 (2004).

${ }^{14}$ M. S. Ferreira, T. G. Dargam, R. B. Muniz, and A. Latgé, Phys. Rev. B 62, 16040 (2000); 63, 245111 (2001).

${ }^{15}$ A. Latgé, C. G. Rocha, L. A. Lavenére, P. Orellana, M. Pacheco, and Z. Barticevic, Phys. Rev. B 67, 155413 (2003).

${ }^{16}$ G. Treboux, P. Lapstun, Z. Wu, and K. Silverbrook, J. Phys. Chem. B 103, 8671 (1999).

${ }^{17}$ K. A. Muttalib and P. Wölfle, Phys. Rev. Lett. 83, 3013 (1999). 